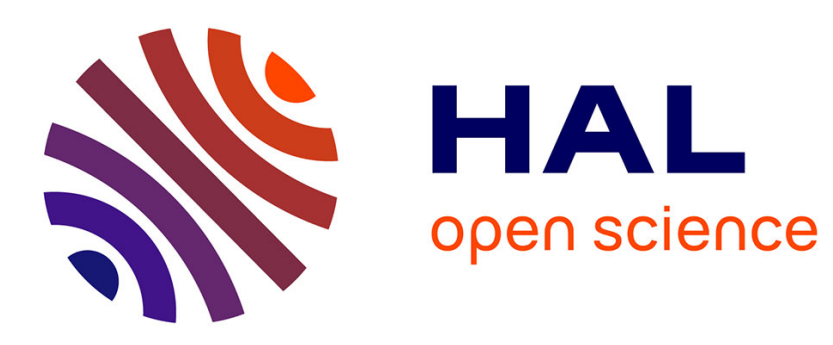

\title{
Continuous Open Source License Compliance
}

Simon Phipps, Stefano Zacchiroli

\section{- To cite this version:}

Simon Phipps, Stefano Zacchiroli. Continuous Open Source License Compliance. Computer, In press, 10.1109/MC.2020.3024403 . hal-03006142

\section{HAL Id: hal-03006142 \\ https://hal.science/hal-03006142}

Submitted on 16 Nov 2020

HAL is a multi-disciplinary open access archive for the deposit and dissemination of scientific research documents, whether they are published or not. The documents may come from teaching and research institutions in France or abroad, or from public or private research centers.
L'archive ouverte pluridisciplinaire HAL, est destinée au dépôt et à la diffusion de documents scientifiques de niveau recherche, publiés ou non, émanant des établissements d'enseignement et de recherche français ou étrangers, des laboratoires publics ou privés. 


\title{
Continuous Open Source License Compliance
}

\author{
Simon Phipps \\ Meshed Insights Ltd, United Kingdom \\ Stefano Zacchiroli \\ Université de Paris and Inria, France
}

\section{Abstract-In this article we consider the role of policy and process in open source usage and propose in-workflow automation as the best path to promoting compliance.}

Freely-licensed open source software (FOSS) has become a key ingredient in software implementation across all sectors and at all scales of enterprise. Once from the realm of informal hacker collaboration enabled by the Internet, it has evolved into a discipline in its own right. Whether approached from the pragmatic angle of OSI-approved licensing or from the ethical angle of user rights, the fundamental enabler of FOSS is the ability of developers to reuse and adapt pre-existing code without the need to seek specific rights-holder approval or negotiate terms. In practice that means it's easy to start at the point of innovation rather than needing to first build (or buy) the well-understood preliminaries and platforms others have already pioneered.

The fact the developer can jump straight to innovation without asking for basic permissions can conceal the fact that FOSS needs managing just like any other software components. It has dependencies that will need to be monitored and updated when there are security exposures; it has a license that grants rights in return for satisfying responsibilities; it comes from a source that needs at least a little ongoing engagement. In this regard, it is just like proprietary software, just with more freedoms.

Of these three management needs, satisfying the responsibilities associated with the (otherwise freely permissive) license is the most pressing.
FOSS can be used only as a result of the grant of licenses to the copyrights and patents its creators have generously offered. Their corresponding requirements thus need respecting if the license is to remain current. The actions associated with this compliance also bring other benefits, such as the creation of a comprehensive manifest for each subsystem (allowing dependency tracking to be performed) and the acknowledgement of the communities and individuals involved. As such, it is both an essential risk management activity and a best practice to keep your code up-to-date and your company in good standing with the communities upon which you depend.

As McAffer [2] explained in this column, these tasks are best performed by a dedicated Open Source Programme Office (OSPO) in larger organisations. But that doesn't mean it all has to be a matter of manual effort! Increasingly, organisations are weaving license compliance into the systems that also continuously build, test and deploy their software. This serves multiple needs:

- The routine hygiene of software for internal use

- The more consequential management of the manifest for software shipped to third parties or embedded in systems

- The specific needs that arise when a company is being prepared to acquire or be acquired. 
While some commercial suppliers tend to emphasise the supposed risks associated with the GNU General Public License (GPL) family, compliance is much broader and more positive than that lens implies. The GPL does indeed require licensees to make the Complete and Corresponding Source code (CCS) available to recipients of the executable code that arises from the use of their licensed materials, but almost every FOSS license has requirements that it's important to respect. Licenses like BSD, MIT and Apache all require a record of the previous contributors to be passed on to users; licenses like MPL and EPL require certain deliverables to be accompanied by source code to varying extents; and so on.

These requirements are all a known quantity with current compliance strategies and most are highly amenable to automation. Doing so controls risk, improves quality and cultivates community respect. In this article, we will thus focus on the current tools and popular workflows that allow FOSS license compliance to be satisfied invisibly most of the time.

\section{THE OPEN SOURCE SUPPLY CHAIN}

Given almost every enterprise software system contains open source software, where has it come from? How is it manipulated and assembled to produce internal systems? Who receives the resulting constructions? These questions define a software supply chain, which may involve a surprisingly long and broad sequence of entities on the inbound side and could include third parties on the outbound side even if your business does not apparently trade in software. A previous column by Harutyunyan [1] covers this concept in more depth.

In brief, your open source supply chain comprises inbound software-the open source and proprietary software entering your enterprisetogether with its dependencies; in-house development, the adaptations you make to inbound software and the software you develop yourself; and then outbound software, the software you pass to others either under open source licenses or under proprietary terms, either as software, as software-implemented services or embedded in hardware.

Managing your open source supply chain will require a comprehensive open source policy.
Fundamentally, your open source policy encapsulates the risks that you consider justified within your business. This will include a determination of which licenses you have analysed and understood, which combinations of those licenses are acceptable within both inbound and outbound software, what management steps are required to ensure the risks are managed and how the determinations are recorded and reviewed.

Your open source policy should go beyond licensing however. You will need policies on how vulnerabilities discovered in inbound software will be evaluated and what steps will be taken to ensure all uses of the affected inbound software are addressed. It is important to have policies on what skills are required in-house for managing critical components and how staff absences/departures will be handled, for example through third-party contractors or through supplier subscriptions. You should also have a policy and associated budget for memberships and sponsorships of community charities (OSI, FSF, Apache etc.) and software trade associations (Eclipse Foundation, Linux Foundation and so on) so that you are able to properly exercise influence as well as make upstream contributions. McAffer has covered governance topics and open source policies in more depth in this series.

These policies will then drive your open source review process. This will be triggered when someone wishes to use inbound software that has not previously been evaluated. Your review will address the needs detailed in your open source policy-including licensing, staffing, maintenance, reputational issues, community influence, component maturity and more. A previous article by Spinellis [4] has covered all the factors that you should take into account when choosing a FOSS component.

At the conclusion of the review, the inbound software will be given a go/no-go determination, usually by your OSPO. The result of the assessment will be recorded so that future use of cleared components does not require any further permission to proceed. This is essential if the core value of open source is to be leveraged.

One further step is the creation of your policy for upstream contribution. Your developers should be able to freely contribute their improvements to the upstream maintainers of your inbound 


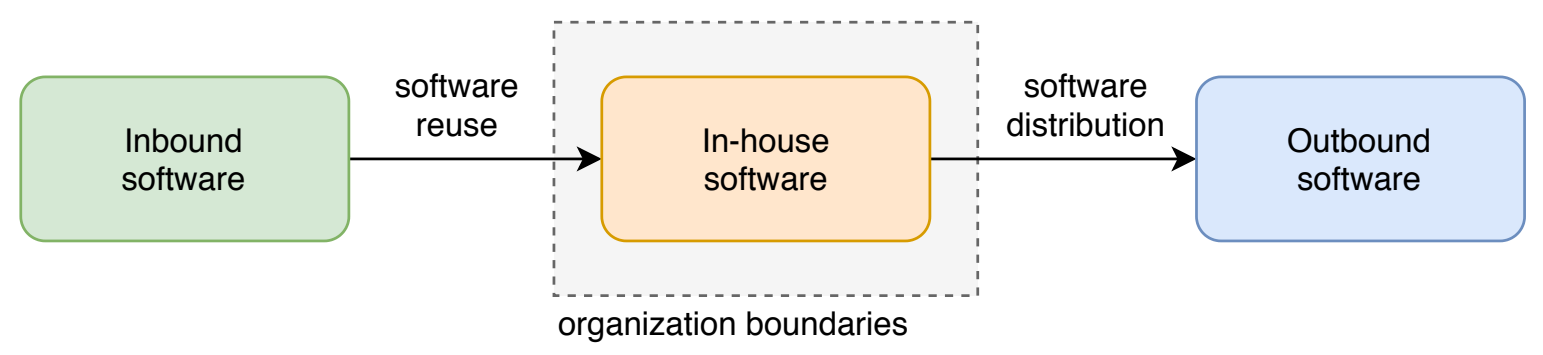

software. Your policy and associated process should ensure that all contributor agreements are reviewed and approved in advance, and that your patent portfolio is not inadvertently used against upstream communities or inbound software.

\section{COMPONENT INVENTORY MANAGEMENT}

Specific tools exist to support the business processes related to open source reviews, in the form of component inventory managers. Eclipse SW360 (https://www.eclipse.org/sw360/) is a popular open source solution in that space and a cornerstone of most enterprise FOSS governance workflows. Let's see how it works, as a classic example of a tool supporting the inbound part of software supply chains.

All software components, open source or otherwise, known to your organization will be added to the organization-specific SW360 instance and assigned a canonical name, allowing to recognize components carrying different names in different contexts (upstream repository, distribution, package manager, etc.) as the same. The source code of component releases will also be uploaded to SW360 and from there automatically analyzed with license scanners, such as FOSSology or ScanCode. Crucially, the addition of novel FOSS components to SW360 can trigger clearance requests, that only specific users (e.g., OSPO members) can perform before the component is deemed fit for use. Once a component is cleared it will remain so for all future uses. Component reviews and clearance decisions are typically based on license scanning results, but can also take into account the other factors we have discussed, such as, known vulnerabilities, development activity, bus factor, etc.

SW360 can also maintain a mapping between the IT products distributed by your organization and the FOSS components they contain, e.g., as dependencies or reused code. This mapping enables SW360 to automatically produce license compliance documents such as Software Bills of Material (SBOM, cf. Managing Your Open Source Supply Chain, previously on this column) in machine-readable standard formats such as SPDX, as well as human-readable documents like the list of all FOSS licenses used in the product, copyright notices for attribution purposes, and offers for source relevant to a given product.

All workflows in SW360 can be performed manually via a web portal or automated via command-line tools and RESTful APIs. You can for instance further support the clearance process by plugging other scanners into the scanning subsystem as well as integrate information retrieved from external knowledge bases, e.g. ClearlyDefined or Software Heritage. The reliance on SPDX as an exchange format allows SW360 to automate the import of entire SBOMs for inbound software, as well as the production of complete and corresponding source (CCS) tarballs for outbound software, when required by the license.

\section{CONTINUOUS LICENSE COMPLIANCE}

Once you have a clear grasp of cleared components in your software supply chain and can fulfill license obligations at a specific point in time, the question that naturally arises is how to maintain that status quo in the long run, as development continues. This boils down to deciding when you review your products for adherence to your internal open source policy.

Various approaches are possible, including sporadic "fire drills", periodic checks, and release-time checks. The best approach is to continuously and automatically check that all your products adhere to your open source policy-this is what we refer to as continuous open source license compliance in this article, or continuous 


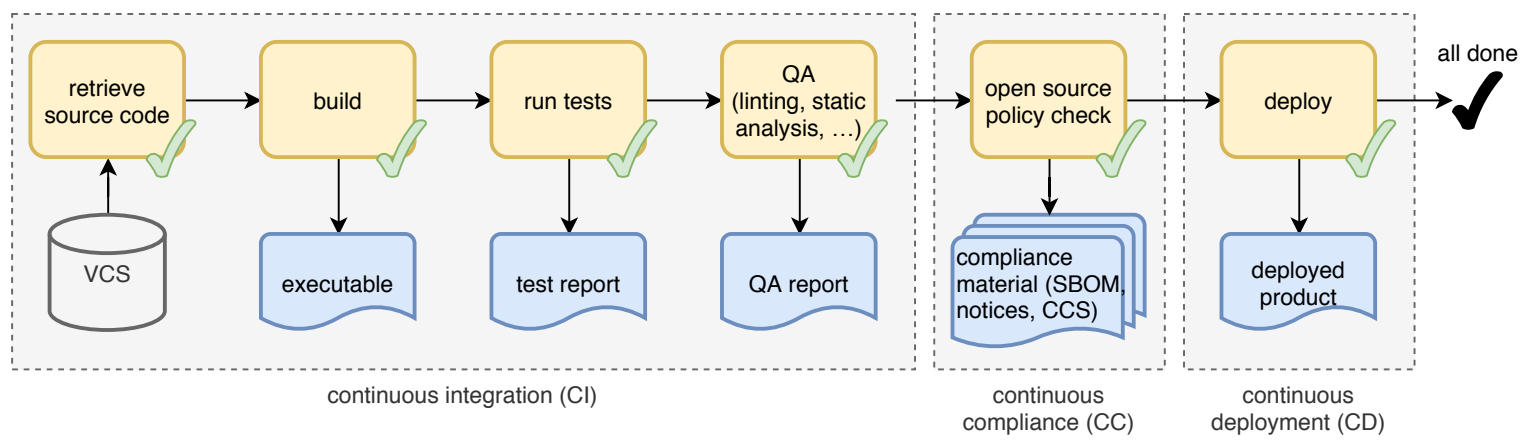

compliance for short.

In practice this means integrating license compliance checks into existing continuous integration/continuous deployment (CI/CD) toolchains, making the build fail in case a divergence between the actual build artifacts and your open source policy is detected. Doing so immediately notifies developers of policy issues that should be dealt with, resulting in shorter feedback loops (a core tenet of agile development practices) and reduced risks of wasted development efforts (e.g., integration of a FOSS component that will need to be replaced before release).

For continuous compliance to work, the verification of adherence to your open source policy should be as automated as possible, so that it stays out of the way of engineers-until things go wrong.

\section{TOOLCHAIN INTEGRATION}

Implementing in practice the general idea of continuous compliance as a fully-automated verification of adherence to your open source policy can be tricky. No one-size-fits-all solution is well-established in the industry yet, nor will probably ever be, due to how each build toolchain is a unique snowflake. Your own implementation of continuous compliance will likely combine several tools and integrate them with custom adapters.

This need is behind the current industry push toward open compliance, i.e., the idea that one should preferably rely on FOSS tools to implement continuous compliance. With FOSS compliance tools it is easier to tailor tools to your specific needs, lock-in risks get mitigated, and you will have opportunities to contribute back to the ecosystem, remaining on top of the technology you use and maintaining influence on software evolution. Proprietary compliance tools can still be used, but are usually integrated as black boxes into toolchains that contain increasingly large majorities of open tools.

The tooling landscape (https://github.com/Open-Source-

Compliance/Sharing-creates-value/) conducted by the Open Source Tooling Group and the OpenChain curriculum (https://github.com/OpenChainProject/curriculum/) provide a good overview of existing tools to support automated governance of FOSS supply chains. As there are too many to be explained here in full, we conclude with a few examples and discuss how they can be used to implement continuous compliance.

Several high-quality license and source code scanners exist. As we have seen, their integration into continuous compliance happens at the component inventory level, during clearance of inbound components. We refer to the comprehensive overview of such tools given by Ombredanne [3] last month on this column for more details.

Dependency trackers ensure that all the dependencies pulled in at build time are known and cleared in your component inventory. This is of paramount importance because, while you can easily audit the direct dependencies declared in your software products, transitive dependencies can change in the ecosystem unexpectedly and might pull in unknown components or newer versions of known components yet to be cleared.

\section{Eclipse SW360 Antenna} (https://www.eclipse.org/antenna/) is a popular tool used to automate dependency tracking that, in conjunction with SW360, constitutes a fairly comprehensive continuous compliance implementation. Antenna integrates with build 
automation tools, like Maven or Gradle, scans source code artifacts of all dependencies during build, verifies that they are cleared in SW360or, alternatively, populates it with them so that they can be cleared later on-and automatically produce compliance material such as the list of all dependencies with their licenses in a spreadsheet-friendly format and a CCS bundle containing all their source code. Integration with other build tools is possible with custom code and the build can be made to fail in case non-cleared components are encountered or if their license does not adhere to your open source policy.

The overall continuous compliance ecosystem is moving at a fast pace, with new tools and approaches being frequently released. Other noteworthy players in the same feature space of what SW360 and Antenna offer are Quartermaster (https://qmstr.org/), hosted by The Linux Foundation as part of their Automated Compliance Tooling (ACT) umbrella project, and the OSS Review Toolkit (ORT, https://github.com/ossreview-toolkit/ort). The latter is particularly interesting as it provides a highly customizable pipeline for continuous compliance, composed of several independent blocks: a dependency analyzer, a downloader for dependencies source code, an abstraction over license scanners, a policy checker that supports custom business rules, and a reporter to build SBOMs. ORT components can, and often are, used independently from the other blocks in custom continuous compliance toolchains.

\section{CONCLUSION}

Continuous open source license compliance is now a well-established industry best practice in managing the life cycle of software products. It consists of automating as much as possible the verification of the adherence of your IT products, that almost invariably contain open source components, to the open source policy of your organization. Ideally, such an automated verification is then integrated into your existing $\mathrm{CI} / \mathrm{CD}$ toolchain, making software builds fail and notifying developers early when issues are spotted.

No one-size-fits-all technology to implement continuous compliance has emerged yet, due to the heterogeneity of build toolchains. On the other hand there is consensus on the types of tools you will need: component inventories, scanners, dependency trackers, policy checkers, and generators of compliance material (SBOMs, notices, and CCS bundles) are all tools of the trade. High quality open source implementations of all these tools exist and should be used as the basis for addressing your specific continuous compliance needs.

\section{REFERENCES}

1. Nikolay Harutyunyan. Managing your open source supply chain-why and how? Computer, 53(6):77-81, 2020.

2. Jeff McAffer. Getting started with open source governance. Computer, 52(10):92-96, 2019.

3. Philippe Ombredanne. Free and open source software license compliance: Tools for software composition analysis. Computer, 53(10):105-109, 2020.

4. Diomidis Spinellis. How to select open source components. Computer, 52(12):103-106, 2019.

Simon Phipps leads the management consultancy he founded, Meshed Insights Ltd, and among other industry roles was head of one of the first staffed OSPOs (at Sun Microsystems). Contact him at: simon@meshedinsights.com.

Stefano Zacchiroli is associate professor of Computer Science at Université de Paris, on leave at Inria. He is the co-founder and СTO of the Software Heritage project. Contact him at zack@irif.fr. 